\title{
Analysis of Harmonic Damping Effect of the Distributed Active Filter System
}

\author{
Po-Tai Cheng Non-member (National Tsing Hua University, Taiwan ptcheng@ee.nthu.edu.tw) \\ Tzung-Lin Lee Non-member (National Tsing Hua University, Taiwan d907904@oz.nthu.edu.tw)
}

Keywords: active filter, distributed active filter system, harmonic resonance, distributed generation

The harmonic resonance in the power distribution system has been reported. The harmonic termination active filter can suppress such resonance phenomenon. However, the harmonic voltage distortion along the line may still be significant due to the harmonics standing wave. This paper proposes the distributed active filter system (DAFS), which consists of multiple active filter units (AFUs) installed at variouslocations along a radial line and a loop line, to provide overall reduction of the harmonic voltage distortion. Each AFU within the DAFS operates like a harmonic conductance as given,

$$
i_{k}^{*}=G_{k}^{*} \cdot E_{h}
$$

where $i_{k}^{*}$ represents the current command of $A F U_{k}$, and $E_{h}$ represents the harmonics components at the installation point of $A F U_{k}$. The DAFS relies on the droop characteristic to coordinate the operations of all AFUs so that the harmonic filtering workload is evenly distributed among AFUs without any communications. The droop characteristic between the conductance command $G_{k}^{*}$ and the voltampere of $A F U_{k}$ is defined as

$$
G_{k}^{*}=G_{k 0}-b_{k} \cdot\left(S_{k 0}-S_{k}\right)
$$

where $G_{k 0}$ is the rated conductance, $b_{k}$ is the droop coefficient, $S_{k 0}$ is the kVA rating, and $S_{k}$ is the volt-ampere consumption.

Fig. 1 and Fig. 2 show the magnifying factor in the radial and loop line. The distributed deployment provides better suppression capability with increasing capacity of AFUs in both circuit configurations, while concentration deployment suffers from the "whack-amole" phenomenon. Therefore, the distribution-deployed AFUs can reduce harmonic voltage more effectively.

Fig. 3 summarizes the harmonic damping performances of various AFUs deployment discussed in this paper. The vertical axis represents the amplitude of the voltage standing wave and the horizontal axis represents the locations along the line. For the radial line model, the natural harmonic resonance occurs at the seventh harmonics. The most suitable deployment is to install $A F U_{1}$ at the end of the radial line for harmonics termination, and $A F U_{2}$ at bus 2 to damp the crest of seventh harmonics. In the loop line model, the natural harmonic resonance occurs at the fifth harmonics. The suitable deployment is to install $A F U_{1}$ and $A F U_{2}$ at bus 5 and bus 3 respectively. The key to deploying AFUs is to break the line into several segments, so that the length of each segment should be shorter than half wavelength of dominant harmonics for most effective damping.

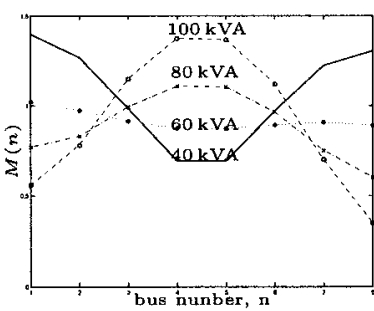

(a) $A F U_{1}, A F U_{2}$ at bus 8

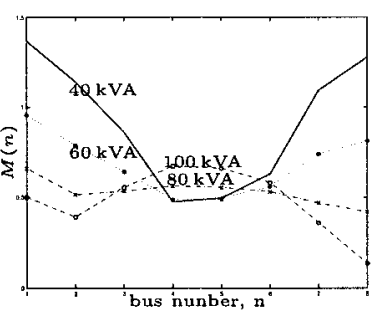

(b) $A F U_{1}$ at bus $8, A F U_{2}$ at bus 2
Fig. 1. The magnifying factor along the radial line

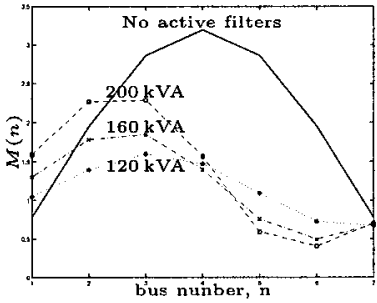

(a) $A F U_{1}, A F U_{2}$ at bus 5 (b) $A F U_{1}$ at bus $5, A F U_{2}$ at bus 3

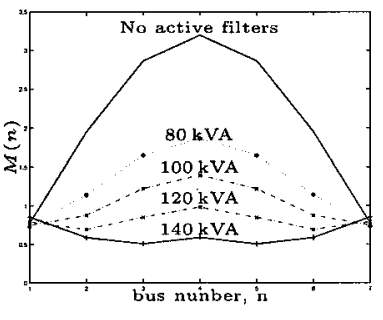

Fig. 2. The magnifying factor along the loop line

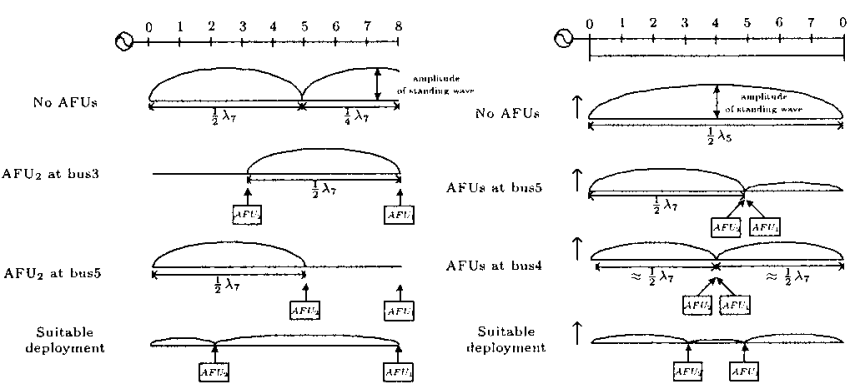

(a) Radial Line

(b) Loop Line

Fig. 3. Deployment strategies of the DAFS and the resulting pattern of harmonic voltages 


\title{
Analysis of Harmonic Damping Effect of the Distributed Active Filter System
}

\author{
Po-Tai Cheng* Non-member \\ Tzung-Lin Lee ${ }^{* *}$ Non-member
}

\begin{abstract}
The harmonic resonance in the power distribution system or in the industrial facilities has been reported. The harmonic termination by installing a shunt active filter at the end of a radial line can suppress harmonic resonance; however, the harmonic voltage distortion along the line may still be significant due to harmonics standing waves. The distributed active filter system, which consists of multiple active filter units deployed along the power line, can effectively reduce voltage harmonics. Based on the pre-determined droop characteristics, the active filter units can share harmonic filtering workload without any communications. In this paper, the distributed active filter system is applied to a radial distribution line and a loop distribution line, which is often used in high-tech industrial parks for enhancing power reliability. This paper provides the theoretic analysis of harmonic resonance, simulation results and laboratory test results to verify the performance of the distributed active filter system. The deployment strategies of active filter units are also presented.
\end{abstract}

Keywords: active filter, distributed active filter system, harmonic resonance, distributed generation

\section{Introduction}

The proliferation of nonlinear loads in power systems has been growing in an unprecedented pace in recent years due to the advance of power electronics technologies. The resulting harmonic pollution in power systems also deteriorates at an alarming rate. Severe voltage distortion due to harmonic resonances has been reported. Previous literatures propose installing a shunt active filter, which operates as a harmonic termination resistor, at the end of a radial line to damp the harmonic resonance ${ }^{(1)-(4)}$. If the active termination resistor is equal to characteristic impedance of the system, the amplification of voltage harmonics will not occur. However, the characteristic impedance of the system often varies and is difficult to identify due to the complexity of distribution systems. Eventually, these factors impede the effectiveness of the harmonic termination active filter in terms of reducing voltage harmonics distortion along the entire line.

The harmonic resonance phenomenon is a mixture of harmonic voltage standing waves of different wavelength ${ }^{(5)}$, so the magnification of harmonic voltages is frequencydependent with maxima and minima occurring at certain locations along the power line. This explains why the harmonic termination active filter at the end of the line can suppress the voltage harmonics of different frequencies along the line only if the termination resistance command of the active filter matches the system characteristic impedance perfectly. The distributed active filter system (DAFS), which consists

\footnotetext{
* Center for Advanced Power Technologies (CAPT), Department of Electrical Engineering, National Tsing Hua University, Hsin-Chu, 30013, Taiwan, ptcheng@ee.nthu.edu.tw

** Center for Advanced Power Technologies (CAPT), Department of Electrical Engineering, National Tsing Hua University, Hsin-Chu, 30013, Taiwan,d907904@oz.nthu.edu.tw
}

of multiple active filter units (AFUs), is proposed by the authors ${ }^{(6)}$. By adequately deploying these AFUs which operate as harmonic resistors along the power line, the magnification of voltage harmonics can be suppressed effectively by dismantling the boundary condition of the standing wave. Furthermore, the AFUs are controlled by a droop characteristic between the harmonic conductance command and the voltampere of each AFU. This feature allows the AFUs to share the harmonic filtering workload without any communications among them. This paper presents the theoretical analysis of harmonic damping of the DAFS in a radial line and a loop line which is often used in high-tech industry park for power reliability enhancement. Computer simulation and laboratory test results are provided to validate the effectiveness of the DAFS.

\section{Analysis of Harmonic Damping}

The harmonic resonance in the distribution system results from the resonance between line inductance and system capacitance. The line inductance is dependent on the leakage inductance of transformers in the system and the stray inductance of the power lines or cables. The system capacitance includes the stray capacitance of power line and power factor correction capacitance within the system. In order to facilitate the harmonic analysis, the line inductances and the system capacitances are assumed to be evenly distributed along the feeder ${ }^{(1)(3)(7)}$. Therefore, an equivalent long line model is established and its parameters are listed in Table 1. For simplification, the propagation constants, wavelengths, and the characteristic impedance are calculated assuming the line is lossless. This paper mainly discusses the harmonic damping capability of the proposed DAFS system at the fifth and the seventh harmonics because high order harmonics seldom excite resonance. 
Table 1. Parameters of a given power line.

\begin{tabular}{ll|ll}
\hline Line voltage & $6.6 \mathrm{kV}$ & Line frequency & $60 \mathrm{~Hz}$ \\
Feeder length, $l$ & $8 \mathrm{~km}$ & Line inductor, $L$ & $2.0 \mathrm{mH} / \mathrm{km}(5.2 \%)$ \\
Line capacitor, $C$ & $28 \mu \mathrm{F} / \mathrm{km}(15.3 \%)$ & Line resistor, $R$ & $0.36 \Omega / \mathrm{km}(2.5 \%)$ \\
Harmonic propagation constant, $\gamma_{5}$ & $j 0.446 \mathrm{~km}^{-1}$ & Harmonic propagation constant, $\gamma_{7}$ & $j 0.624 \mathrm{~km}^{-1}$ \\
Wavelength of the fifth harmonics, $\lambda_{5}$ & $14.09 \mathrm{~km}$ & Wavelength of the seventh harmonics, $\lambda_{7}$ & $10.06 \mathrm{~km}$ \\
Characteristic impedance, $z$ & $8.45 \Omega$ & & \\
\hline
\end{tabular}

Most harmonic producing nonlinear loads are dispersed along a power line so the harmonic voltage is not easy to analyze and formulate. However, the harmonic voltage is the vector sum of individual component induced by different harmonic source according to superposition theorem so a single harmonic voltage source is considered in this paper. In order to assess the amplification of voltage harmonics, a magnifying factor $M_{h}$ along the power line is defined as follows:

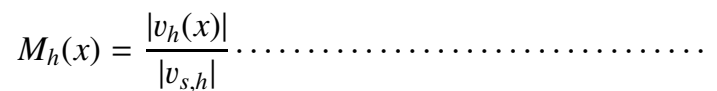

where the suffix $h$ denotes the order of harmonics, $v_{h}(x)$ is the harmonic voltage at position $x$, and $v_{s, h}$ is the harmonic voltage source. In the following, active filters operating as a harmonic conductance are installed at in the radial and loop lines to investigate the phenomenon of harmonic resonance.

2.1 Radial Line Model The voltage at a certain location is the vector sum of all forward waves and reflected waves of a finite transmission line ${ }^{(5)}$. The magnitude and phase of the reflected wave are primarily determined by the matching condition between the line and the terminator. If the incident wave is in phase with the reflected wave, the resulting voltage oscillation will be very severe due to the full reflection. The condition of full reflection for a radial line is given as:

$$
l=n \cdot \lambda / 4 \text {. }
$$

where $l$ represents the length of radial line, $\lambda$ is the wavelength, and $n$ is an arbitrary odd positive integer. If this condition holds, a periodically magnifying phenomenon of harmonic voltage will occur along the radial line.

Fig. 1 shows the distributed-parameter model of a radial line at harmonic frequencies with two active filters installed at different locations: $A F U_{1}$ at the end of line and $A F U_{2}$ at a distance of $l_{1}$ from source. $G_{1}$ and $G_{2}$ are the conductance commands of $A F U_{1}$ and $A F U_{2}$ respectively. Because the transmission line is divided into two sections by $A F U_{2}$, two sets of boundary equations must be combined. The harmonic voltage at position $x$ can then be expressed as:

$$
v_{h}(x)=\left\{\begin{array}{l}
\frac{z_{2} \cosh \gamma_{h}\left(l_{1}-x\right)+z \sinh \gamma_{h}\left(l_{1}-x\right)}{z_{2} \cosh \gamma_{h} l_{1}+z \sinh \gamma_{h} l_{1}} v_{s, h} \\
\begin{array}{rl}
\frac{1}{G_{1}} \cosh \gamma_{h}(l-x)+z \sinh \gamma_{h}(l-x) & 0<l_{1} \\
\frac{1}{G_{1}} \cosh \gamma_{h}\left(l-l_{1}\right)+z \sinh \gamma_{h}\left(l-l_{1}\right) & v_{h}\left(l_{1}\right) \\
l_{1}<x<l
\end{array}
\end{array}\right.
$$

where the definitions of the above variables are given in Appendix 1. Fig. 2(a) and Fig. 2(b) show the simulation results with $A F U_{1}$ and $A F U_{2}$ installed at $x=8 \mathrm{~km}$ and $x=4 \mathrm{~km}$ respectively, where $G_{1}=G_{2}$ is assumed for simplification. Because the feeder length is approximately three-fourth wavelength of the seventh harmonic and half wavelength of the

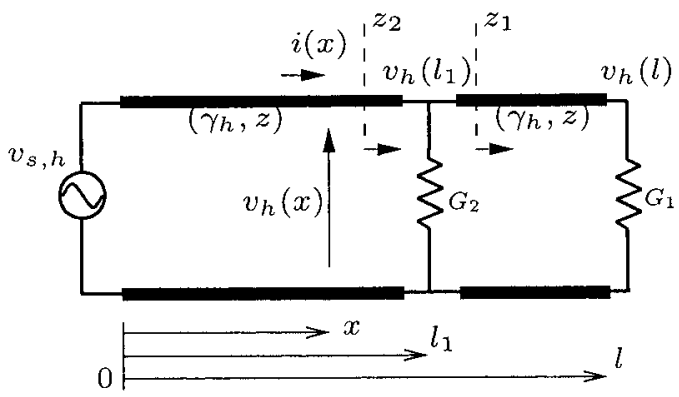

Fig. 1. The distributed-parameter model of a radial line at harmonic frequencies

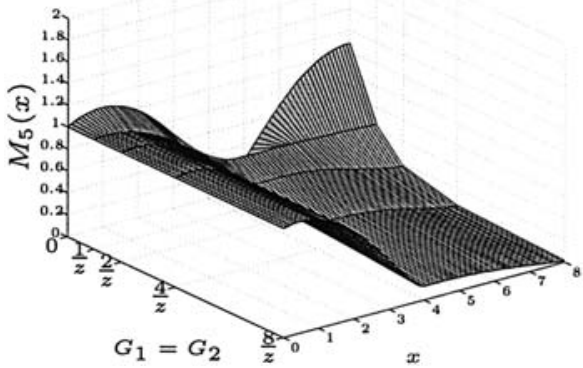

(a) The magnifying factor for the fifth harmonic

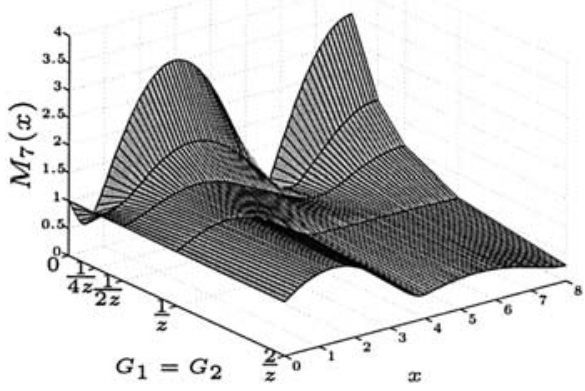

(b) The magnifying factor for the seventh harmonic

Fig. 2. The magnifying factor along the radial line with $A F U_{1}$ at $x=8 \mathrm{~km}$ and $A F U_{2}$ at $x=4 \mathrm{~km}$

fifth harmonic, the so-called "whack-a-mole" phenomenon occurs if a single termination active filter is installed at the end of the radial line. This termination active filter may suppress the seventh harmonic resonance successfully but unintentionally gives rise to the fifth harmonic resonance. Wada et al. have presented detailed discussions on the "whack-amole" phenomenon ${ }^{(3)}$. However, the "whack-a-mole" phenomenon between $M_{5}(x)$ and $M_{7}(x)$ no longer exists in the distributed installation of two AFUs as illustrated in Fig. 2(a) and Fig. 2(b). The reason is that the power line is divided into two sections by $A F U_{2}$, and each section is too short to sustain any significant standing wave at the fifth and the 


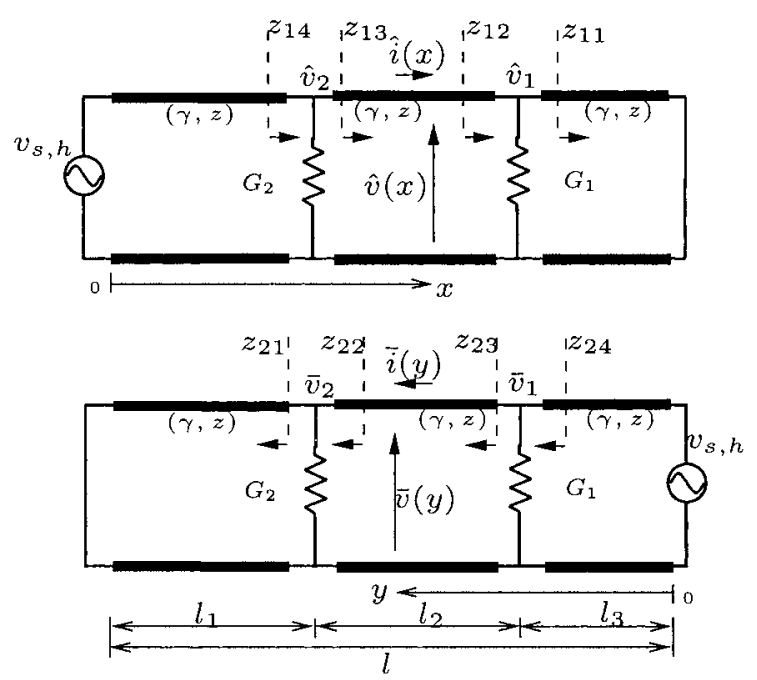

Fig. 3. The equivalent distributed-parameter model of a loop line at harmonic frequencies

seventh harmonic frequencies. Compared to the harmonic termination active filter, the distributed active filters suppress the voltage harmonics more effectively.

2.2 Loop Line Model For simplicity, the loop distribution line is configured by joining two ends of radial line together. The resonance mechanism in the loop line is quite different from that in the radial line. The condition of existence for the standing wave is given as,

$$
l=n \cdot \lambda / 2
$$

where $l$ represents the length of the line and $n$ is an odd positive integer. The harmonic voltage standing wave can be derived by superimposing the two distributed-parameter models in Fig. 3. The harmonic voltage at point $x$ from the source can be expressed as

$$
v(x)=\hat{v}(x)+\bar{v}(l-x)
$$

where

$$
\begin{aligned}
& \hat{v}(x)=\left\{\begin{array}{lr}
\frac{\hat{z}_{14} \cosh \gamma_{h}\left(l_{1}-x\right)+z \sinh \gamma_{h}\left(l_{1}-x\right)}{\hat{z}_{14} \cosh \gamma_{h} l_{1}+z \sinh \gamma_{h} l_{1}} v_{s, h} & 0<x<l_{1} \\
\frac{\hat{z}_{12} \cosh \gamma_{h}\left(l_{1}+l_{2}-x\right)+z \sinh \gamma_{h}\left(l_{1}+l_{2}-x\right)}{\hat{z}_{12} \cosh \gamma_{h} l_{2}+z \sinh \gamma_{h} l_{2}} \hat{v}_{2} & l_{1}<x<l_{1}+l_{2} \\
\frac{\sinh \gamma_{h}(l-x)}{\sinh \gamma_{h} l_{3}} \hat{v}_{1} & l_{2}+l_{3}<x<l
\end{array}\right. \\
& \bar{v}(y)=\left\{\begin{array}{lr}
\frac{\bar{z}_{24} \cosh \gamma_{h}\left(l_{3}-y\right)+z \sinh \gamma_{h}\left(l_{3}-y\right)}{\bar{z}_{24} \cosh \gamma_{h} l_{1}+z \sinh \gamma_{h} l_{1}} v_{s, h} \\
0<y<l_{3} \\
\frac{\bar{z}_{22} \cosh \gamma_{h}\left(l_{2}+l_{3}-y\right)+z \sinh \gamma_{h}\left(l_{2}+l_{3}-y\right)}{\bar{z}_{22} \cosh \gamma_{h} l_{2}+z \sinh \gamma_{h} l_{2}} \bar{v}_{1} \\
l_{3}<y<l_{2}+l_{3} \\
\frac{\sinh \gamma_{h}(l-x)}{\sinh \gamma_{h} l_{1}} \bar{v}_{2} \quad l_{2}+l_{3}<y<l
\end{array}\right.
\end{aligned}
$$

The definitions of the above parameters are given in Appendix 2. Clearly, the amplitude of the resonance is affected by the harmonic conductance command and the point of installation of the active filter units. Fig. 4(a) shows that $M_{5}(x)$ is severely amplified if no AFUs are used $\left(G_{1}=G_{2}=0\right)$

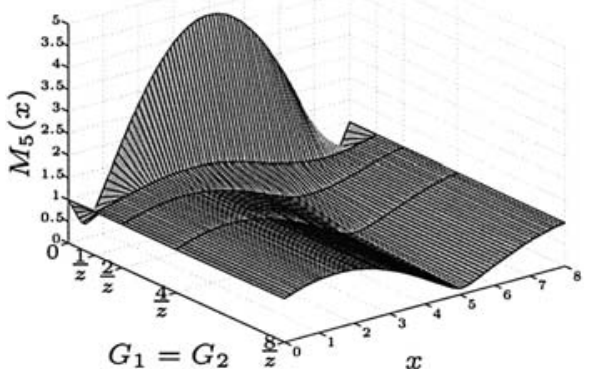

(a) The magnifying factor for the fifth harmonic

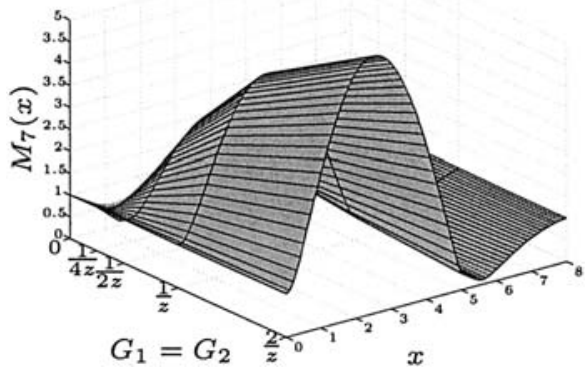

(b) The magnifying factor for the seventh harmonic

Fig. 4. The magnifying factor along the loop line if both $A F U_{1}$ and $A F U_{2}$ are installed at $x=5 \mathrm{~km}$

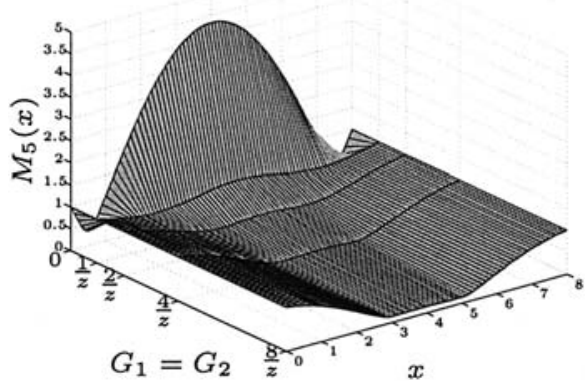

(a) The magnifying factor for the fifth harmonic

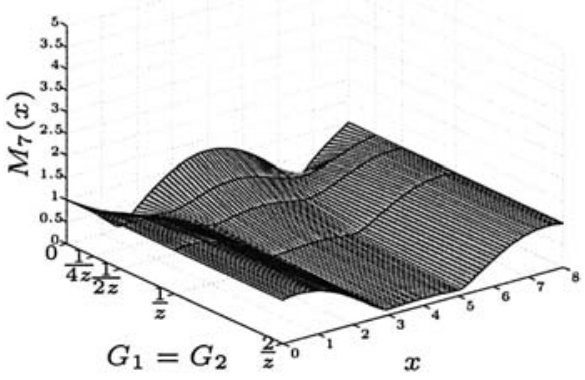

(b) The magnifying factor for the seventh harmonic

Fig. 5. The magnifying factor along the loop line if $A F U_{1}$ and $A F U_{2}$ are installed at $x=5 \mathrm{~km}$ and $x=3 \mathrm{~km}$ respectively

because $l \approx \lambda_{5} / 2$ sustains the fifth harmonic resonance. With both $A F U_{1}$ and $A F U_{2}$ installed at $x=5 \mathrm{~km}$, the line is divided into a $3 \mathrm{~km}$ segment and a $5 \mathrm{~km}$ segment. $M_{5}(x)$ is 
suppressed by the AFUs as illustrated in Fig. 4(b). But $M_{7}(x)$ increases significantly in the $5 \mathrm{~km}$ segment because its length is very close to $\lambda_{7} / 2(=5.03 \mathrm{~km})$. If $A F U_{1}$ and $A U F_{2}$ are installed at $x=5 \mathrm{~km}$ and $x=3 \mathrm{~km}$ respectively, then all segments divided by AFUs are shorter than $\lambda_{5} / 2$ and $\lambda_{7} / 2$. The standing waves at the fifth and seventh harmonics no longer occur. Fig. 5 shows that both $M_{5}(x)$ and $M_{7}(x)$ are subdued by AFUs. Therefore, distributed installation of active filters divides the loop line into small segments. The harmonic resonance can be effectively suppressed if these segments are shorter than half wavelength of dominant harmonics. Compared to the concentrated deployment, the distributed deployment of active filters can reduce the voltage harmonics more effectively.

\section{Damping Performances of DAFS}

Fig. 6 shows the control block diagram of each individual AFU of the DAFS proposed by the authors ${ }^{(6)}$. AFUs are implemented by conventional voltage source inverters as shown in Fig. 7. Each AFU operates like a harmonic conductance as given,

$$
i_{q d}^{e *}=G^{*} \cdot \tilde{E}_{q d}^{e}
$$

where $i_{q d}^{e *}$ represents the current command of the AFU, $\tilde{E}_{q d}^{e}$ represents the harmonic components of the voltage at the AFU installation point, and $G^{*}$ is the conductance command of the AFU. The control of each AFU is implemented in the synchronous reference frame. Three phase voltages $E_{a, b, c}$ are measured, and transformed into the synchronous reference frame. The AC component in the synchronous reference frame represents the harmonic component, so the harmonic voltage components $\tilde{E}_{q d}^{e}$ can be extracted by high-pass filter (HPF). Beside, volt-ampere consumption $S$ can be calculated according to the voltage at the installation point and current consumption of AFU. The droop controller then determines the harmonic conductance command. The current command of the AFU is calculated based on the voltage harmonics $\tilde{E}_{q d}^{e}$ and the harmonic conductance command $G^{*}$ as in (5). The current command is then synthesized by the current regulator and space vector PWM to allow the harmonic filtering functionality of the AFU.
The droop characteristic between the conductance command $G^{*}$ and the volt-ampere of the AFU $S$ is defined as:

$$
G^{*}=G_{0}-b \cdot\left(S_{0}-S\right) \cdot
$$

where $G_{0}$ is the rated conductance and $b$ is the droop coefficient. The kVA rating of the AFU, $S_{0}$, is defined as the product of line voltage RMS value and inverter current RMS value. $S$ represents volt-ampere consumption of the AFU. The DAFS relies on the droop characteristic to coordinate the operations of AFUs so that the harmonic filtering workload is evenly distributed among AFUs without any communications. In this section, the DAFS is applied to the radial line and the loop line to demonstrate its functionality in suppressing harmonic resonance and to evaluate proper locations of AFU deployment.

3.1 Radial Line The radial line is divided into eight segments with nine buses, bus 0 to bus 8 . The harmonic voltage source is at bus $0 . A F U_{1}$ is installed at bus 8 to prevent the full reflection, while $A F U_{2}$ is deployed at various buses along the line. To evaluate the harmonic damping performance of the DAFS, the magnifying factor $M(n)$ at the bus $n$ and average magnifying factor $M_{\text {avg }}$ of the entire line are defined as

$$
M(n)=\sqrt{\frac{\sum_{h} v_{h}^{2}(n)}{\sum_{h} v_{s, h}^{2}}} ; \quad M_{\text {avg }}=\frac{1}{8} \sum_{n=1}^{8} M(n) \cdots \cdots
$$

where $n$ represents the number of buses. Note that the harmonic voltage source is placed at bus 0 for this calculation, and the magnifying factor at bus 0 is always one whether AFUs operate or not. Therefore, bus 0 is excluded from the calculation of average magnifying factor. In the following tests, the harmonic voltage source is assumed to be $0.1 \mathrm{pu}$ at the fifth harmonic frequency and $0.1 \mathrm{pu}$ at the seventh harmonic frequency. Fig. 8 shows average magnifying factor of the line when $A F U_{1}$ is at bus 8 and $A F U_{2}$ is at different buses. If both AFUs are at bus $8, M_{\text {avg }}$ decreases from 1.1 to 0.9 as the capacity of each AFU increases from $40 \mathrm{kVA}$ to $100 \mathrm{kVA}$. Although $M_{a v g}$ may seem under control in this case, the harmonic resonance occurs on the remaining buses as illustrated in Fig. 9(a). The only exception is when the capacities of

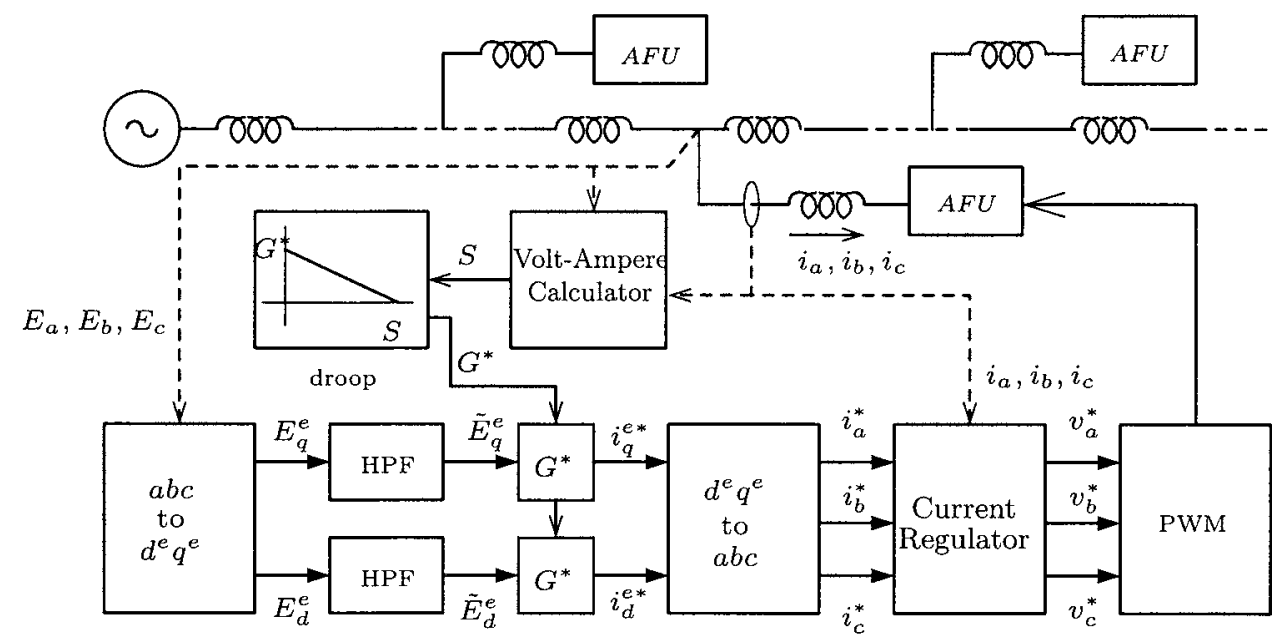

Fig. 6. The control block diagram of the active filter unit (AFU) within the distributed active filter system (DAFS) 
AFUs are $60 \mathrm{kVA}$, where the equivalent conductance of the AFUs is very close to the characteristic impedance of the radial line. Therefore, installing both AFUs at the end of the radial line is not adequate to damp the harmonic resonance. Fig. 8 shows that $A F U_{2}$ at bus 2 can reduce $M_{\text {avg }}$ more effectively than $A F U_{2}$ at other buses. Fig. 9(b) also shows that the damping effect of this arrangement improves as the capacity of AFUs increases. From the evaluation of $M_{\text {avg }}$ in Fig. 8, the deployment of $A F U_{2}$ at bus 2 and $A F U_{1}$ at bus 8 provides more effective harmonic damping for this radial line considering various filtering capacities.

The main strategy of DAFS deployment is to place $A F U_{1}$ at the end of the radial line to terminate the full reflection, and $A F U_{2}$ at the other wave crest of $M_{7}(n)$ or $M_{5}(n)$ to cooperatively damp the harmonics. Howerver, the location of $A F U_{2}$ should avoid the following situations:

$$
\bar{l}=m \cdot \lambda_{5} / 2 \quad \text { or } \quad \bar{l}=m \cdot \lambda_{7} / 2 \text {. }
$$

where $\bar{l}$ represents the length of line segments divided by $A F U_{2}$ and $A F U_{1}$, and $m$ is an arbitrary positive integer. The segment length of (8) could establish standing wave of the fifth or the seventh harmonics, therefore the location of $A F U_{2}$

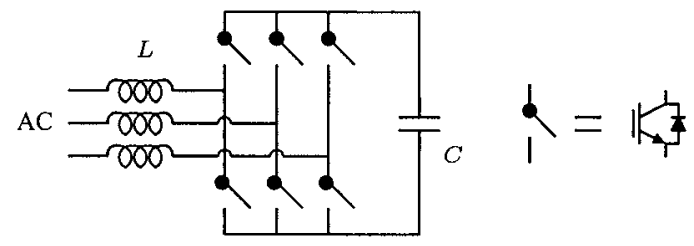

Fig. 7. The inverter of the active filter unit

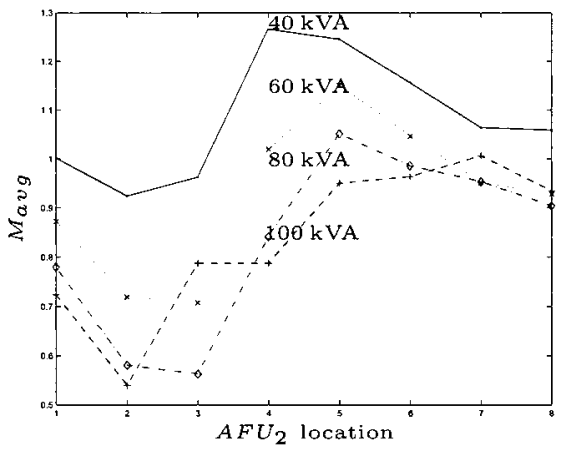

Fig. 8. The average magnifying factor for various installation locations of $A F U_{2}$ with different VA capacities when $A F U_{1}$ is at bus 8 should avoid such scenario.

In this radial line, reducing $M_{7}(n)$ is the primary assignment of the DAFS because the length of the radial line $(8 \mathrm{~km})$ is very close to $3 \lambda_{7} / 4=7.5 \mathrm{~km}$. The first wave crest is at $\lambda_{7} / 4=2.5 \mathrm{~km}$ from the harmonics source $v_{s, h}$, so bus 2 and bus 3 are suitable locations for $A F U_{2}$. From (8), the standing wave may occur if $A F U_{2}$ is placed at bus 3 or bus 6 because such installations create line segments of $\lambda_{7} / 2$ (close to $5 \mathrm{~km}$ ). Thus these locations must be avoided. In addition, line segments of $\lambda_{5} / 2(\approx 7 \mathrm{~km})$ should also be avoided to prevent the fifth harmonic voltage standing wave, therefore buses 1, 7, and 8 are not suitable locations for $A F U_{2}$. From the above observations, bus 2 is the most suitable location for $A F U_{2}$. $M_{a v g}$ in Fig. 8 also validates this installation point.

3.2 Loop Line In the loop line, the concentrated deployment of active filters may result in the "whack-a-mole" phenomenon as mentioned in Sec. 2.2. By deploying multi-

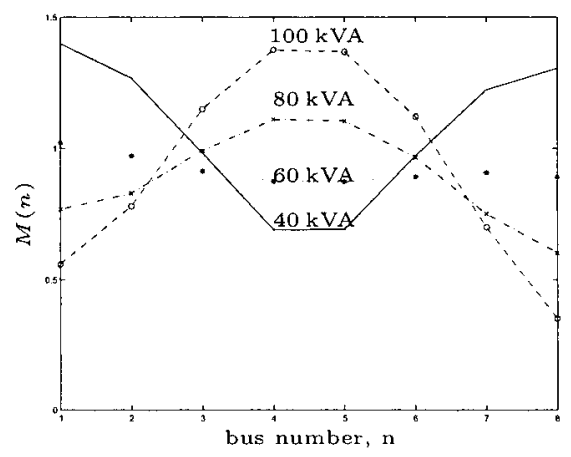

(a) $A F U_{1}, A F U_{2}$ at bus 8

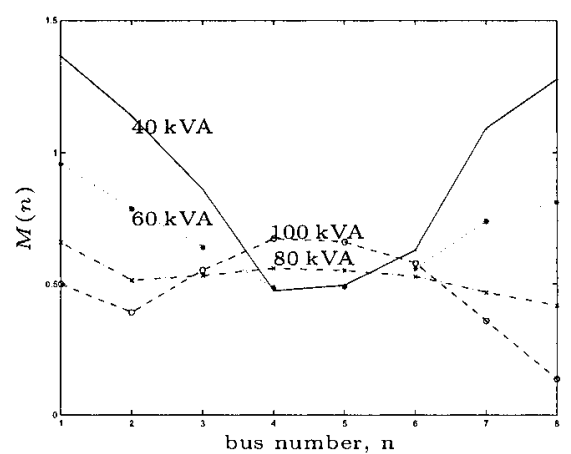

(b) $A F U_{1}$ at bus $8, A F U_{2}$ at bus 2

Fig. 9. The total magnifying factor for two deployments of AFUs with different VA capacities in the radial line

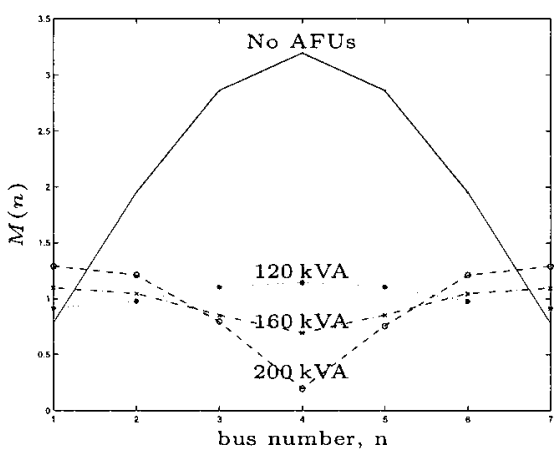

(a) $A F U_{1}, A F U_{2}$ at bus 4

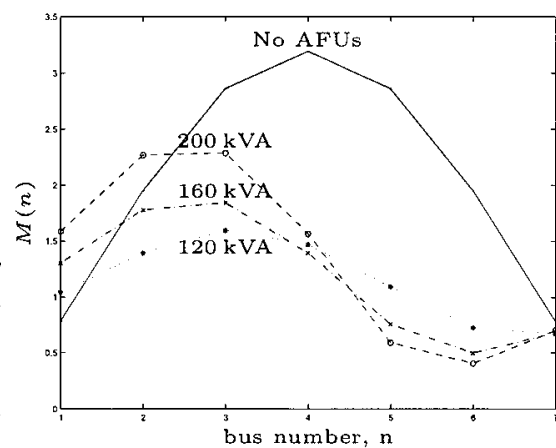

(b) $A F U_{1}, A F U_{2}$ at bus 5

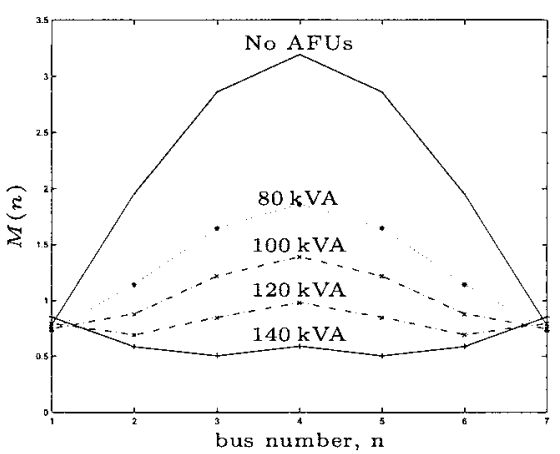

(c) $A F U_{1}$ at bus $5, A F U_{2}$ at bus 3

Fig. 10. The total magnifying factor for various deployments of AFUs with different VA capacities in the loop line 


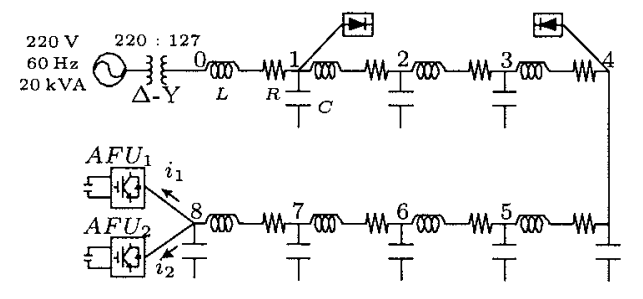

(a) $A F U_{1}, A F U_{2}$ at bus 8

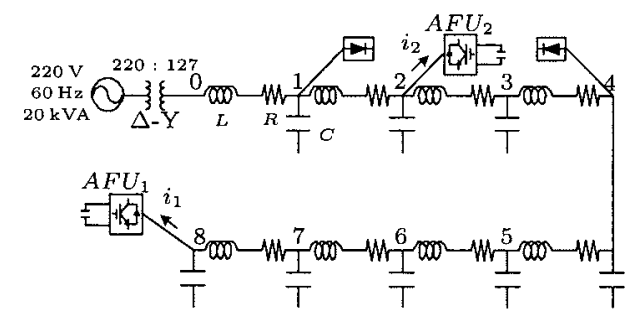

(b) $A F U_{1}$ at bus $8, A F U_{2}$ at bus 2

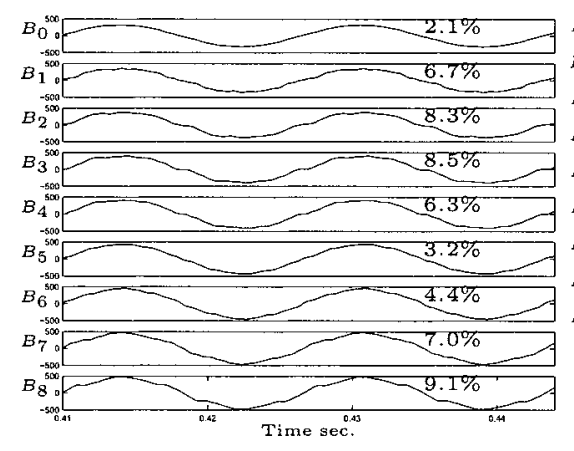

(c) Bus voltages when AFUs are off

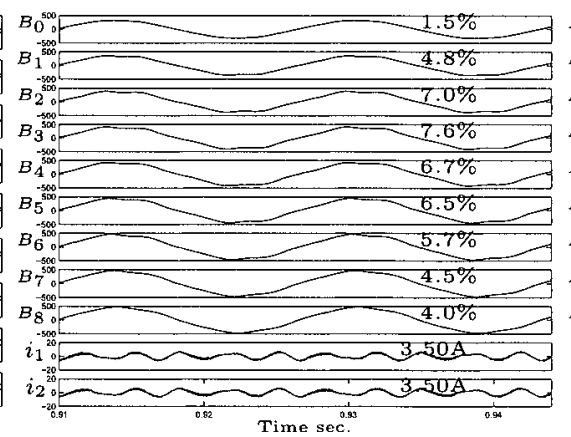

(d) Bus voltages and AFUs currents in Fig. 11(a)

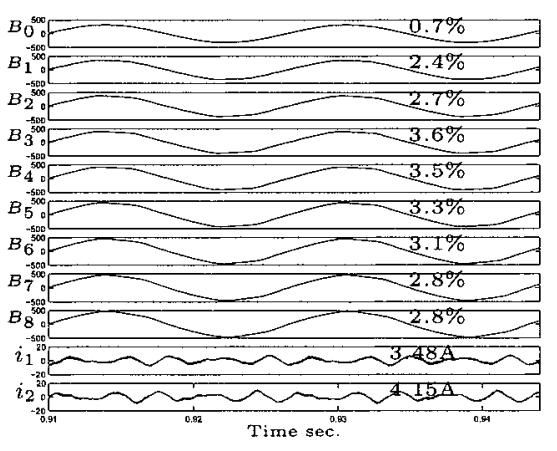

(e) Bus voltages and AFUs currents in Fig. 11(b)

Fig. 11. Simulation circuits and results in the radial line

ple AFUs, the proposed DAFS can break the loop into small segments which are shorter than half wavelength of dominant harmonics, thus preventing the standing wave and the harmonic resonance. In this section, different deployments of active filters are evaluated to examine the damping capability of the DAFS.

Before AFUs are started, Fig. 10 shows that the amplification of $M(n)$ is severe especially at bus 4 because $\lambda_{5} / 2=7 \mathrm{~km}$ is close to the length of line. If both AFUs are at bus 4, M(4) at bus 4 is clearly reduced, but the damping is not so effective for the remaining buses as shown in Fig. 10(a). Fig. 10(b) shows test results of both AFUs at bus 5. M(5) at bus 5 decreases significantly due to the AFUs operation. However, the harmonics is amplified near bus 2 and bus 3 because the $5 \mathrm{~km}$ segment created by this AFUs deployment is equal to $\lambda_{7} / 2$. Such amplification can not be reduced even with increasing AFUs capacities.

Fig. 10(c) shows the test results with $A F U_{1}$ at bus 5 and $A F U_{2}$ bus 3. $M(n)$ is effectively suppressed on all buses, and the effectiveness grows with increasing capacities of AFUs. This deployment divides the loop into segments of $3 \mathrm{~km}$, $2 \mathrm{~km}$ and $3 \mathrm{~km}$ respectively, so standing waves of the fifth or the seventh voltage harmonics can not be established.

\section{Time-domain Simulation Results}

Circuit models of the radial line and the loop line are implemented on the alternative transient program (ATP) platform to verify the harmonic damping capability of the proposed DAFS and the load sharing functionality of the proposed $G-S$ droop control method. The parameters in the simulation are given as follows,

- Power system: $220 \mathrm{~V}$ (line-to-line), $60 \mathrm{~Hz}, 20 \mathrm{kVA}$. The lumped circuit parameters are $L=0.4 \mathrm{mH}(6.2 \%), R=$ $0.0125 \Omega(0.5 \%), C=150 \mu \mathrm{F}(13.7 \%)$.

- Nonlinear loads: Two diode rectifiers with filter inductor, DC capacitor, and load resistor are rated at $2760 \mathrm{VA}$ and 3348 VA respectively.

- Active filters: The frequency of PWM operation is $10 \mathrm{kHz}$. The droop parameters are $G_{0}=0 \Omega^{-1}$, and $b_{1}=b_{2}=-1 \times 10^{-3} V^{-2}$.

4.1 Radial Line In this section, the damping performance of both termination installation and distributed installation of AFUs in the radial line are evaluated. The impact of the kVA rating of AFUs on the damping ability is also discussed.

Fig. 11(a) shows the lumped circuit model of long distribution line ${ }^{(3)(4)} . A F U_{1}$ and $A F U_{2}$ rated at $2.0 \mathrm{kVA}$ each are installed at bus 8 to test the effectiveness of the harmonic termination active filter. Before the AFUs are started, the harmonic distortion is very severe due to the harmonic amplification along the radial line, as shown in Fig. 11(c). After the AFUs are started, the THDv on bus 8 is significantly reduced from $9.1 \%$ to $4.0 \%$ as illustrated in Fig. 11(d). But THDv on middle buses become worse, for example, THDv on bus 5 increases from $3.2 \%$ to $6.5 \%$ due to the standing wave of the $5^{\text {th }}$ harmonics. At steady state, $A F U_{1}$ and $A F U_{2}$ reach $G_{1}=G_{2}=0.490 \Omega^{-1}$ based on the droop characteristics, and both units absorb $3.50 \mathrm{~A}$ (RMS) of harmonic current and consume $1944 \mathrm{VA}$.

In Fig. 11(b), $A F U_{1}$ is installed at bus 8 and $A F U_{2}$ is at bus 2 to test the effectiveness of distributed installation of AFUs. The waveforms in Fig. 11(e) show that THDv are improved even further compared to Fig. 11(d), THDv on the middle buses are suppressed effectively because the distributed deployment of AFUs suppresses the standing wave. The $G-S$ droop characteristics also allows the AFUs to share the filtering workload evenly. At steady state, $A F U_{1}$ and $A F U_{2}$ consume $1909 \mathrm{VA}$ and $1935 \mathrm{VA}$ respectively with conductance commands $G_{1}=0.903 \Omega^{-1}$ and $G_{2}=0.734 \Omega^{-1}$.

Fig. 12 shows the THDv on all buses if AFUs with different capacities are applied to the test scenarios of Fig. 11(a) and Fig. 11(b). Fig. 12(b) shows that larger capacities of AFUs 


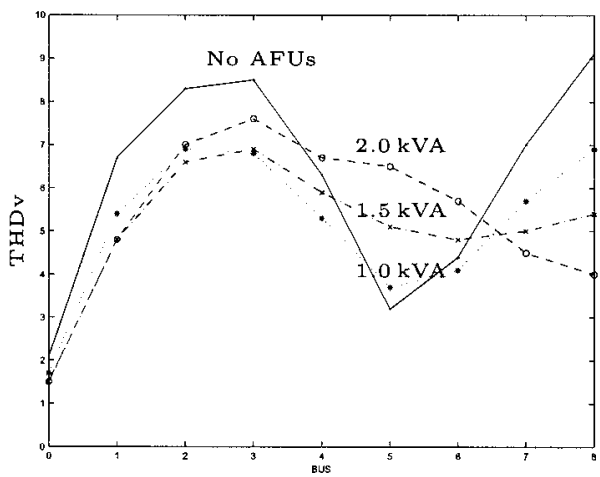

(a) $\mathrm{AFU}_{1}, \mathrm{AFU}_{2}$ at bus 8

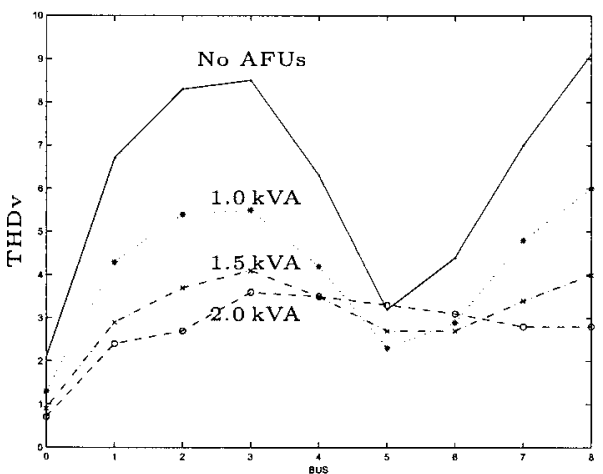

(b) $\mathrm{AFU}_{1}$ at bus $8, \mathrm{AFU}_{2}$ at bus 2

Fig. 12. THDv along the radial line in Fig. 11(a) and Fig. 11(b) for AFUs of various capacities

Table 2. Statistics of THDv along the radial line when $\mathrm{AFU}_{1}$ is at bus 8 and $\mathrm{AFU}_{2}$ is at various installation locations

\begin{tabular}{|c|c|c|c|c|c|c|c|c|c|c|}
\hline Bus number & 0 & 1 & 2 & 3 & 4 & 5 & 6 & 7 & 8 & Average THDv \\
\hline$A F U_{1}$ at $8, A F U_{2}$ at 1 & $0.8 \%$ & $2.5 \%$ & $4.3 \%$ & $4.8 \%$ & $4.1 \%$ & $3.5 \%$ & $3.3 \%$ & $3.6 \%$ & $4.1 \%$ & $3.44 \%$ \\
\hline$A F U_{1}$ at $8, A F U_{2}$ at 2 & $0.7 \%$ & $2.4 \%$ & $2.7 \%$ & $3.6 \%$ & $3.5 \%$ & $3.3 \%$ & $3.1 \%$ & $2.8 \%$ & $2.8 \%$ & $2.76 \%$ \\
\hline$A F U_{1}$ at $8, A F U_{2}$ at 3 & $1.0 \%$ & $3.1 \%$ & $3.5 \%$ & $3.3 \%$ & $3.3 \%$ & $3.2 \%$ & $2.9 \%$ & $2.4 \%$ & $2.1 \%$ & $2.76 \%$ \\
\hline$A F U_{1}$ at $8, A F U_{2}$ at 4 & $1.1 \%$ & $3.6 \%$ & $4.5 \%$ & $4.3 \%$ & $3.4 \%$ & $3.3 \%$ & $3.0 \%$ & $2.6 \%$ & $2.4 \%$ & $3.13 \%$ \\
\hline$A F U_{1}$ at $8, A F U_{2}$ at 5 & $1.7 \%$ & $5.4 \%$ & $7.0 \%$ & $6.8 \%$ & $5.1 \%$ & $3.4 \%$ & $3.2 \%$ & $3.3 \%$ & $3.7 \%$ & $4.40 \%$ \\
\hline$A F U_{1}$ at $8, A F U_{2}$ at 6 & $1.8 \%$ & $5.8 \%$ & $7.5 \%$ & $7.6 \%$ & $6.6 \%$ & $4.7 \%$ & $3.1 \%$ & $3.3 \%$ & $3.4 \%$ & $4.87 \%$ \\
\hline$A F U_{1}$ at $8, A F U_{2}$ at 7 & $1.7 \%$ & $5.5 \%$ & $7.8 \%$ & $8.4 \%$ & $7.3 \%$ & $5.9 \%$ & $4.3 \%$ & $3.6 \%$ & $4.0 \%$ & $5.39 \%$ \\
\hline$A F U_{1}$ at $8, A F U_{2}$ at 8 & $1.5 \%$ & $4.8 \%$ & $7.0 \%$ & $7.6 \%$ & $6.7 \%$ & $6.5 \%$ & $5.7 \%$ & $4.5 \%$ & $4.0 \%$ & $5.36 \%$ \\
\hline No AFUs & $2.1 \%$ & $6.7 \%$ & $8.3 \%$ & $8.5 \%$ & $6.3 \%$ & $3.2 \%$ & $4.4 \%$ & $7.0 \%$ & $9.1 \%$ & $6.18 \%$ \\
\hline
\end{tabular}

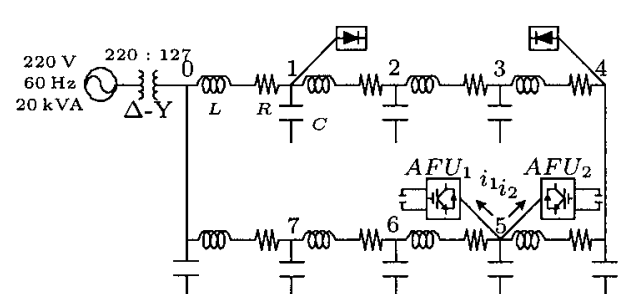

(a) $A F U_{1}, A F U_{2}$ at BUS 5

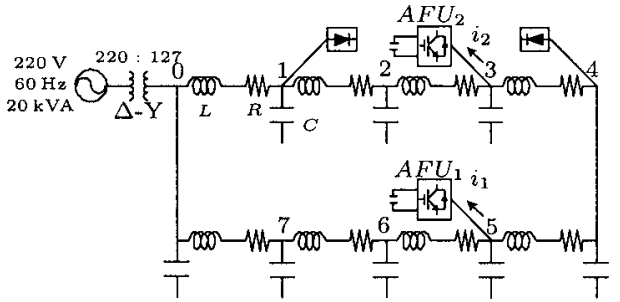

(b) $A F U_{1}$ at BUS 5, $A F U_{2}$ at BUS 3

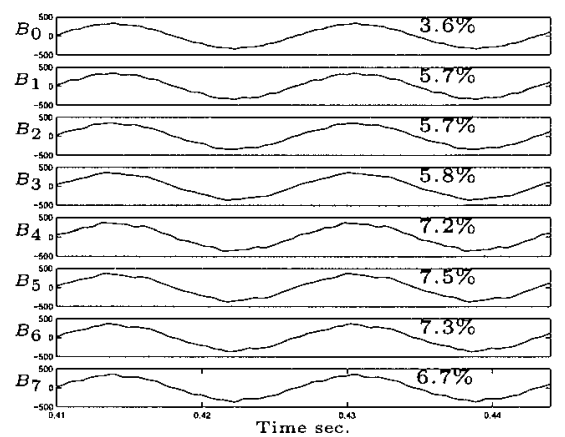

(c) Bus voltage waveforms when AFUs are off

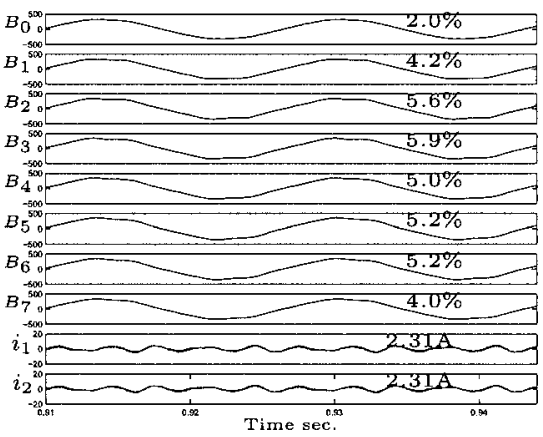

(d) Bus voltages and AFUs currents in Fig. 13(a)

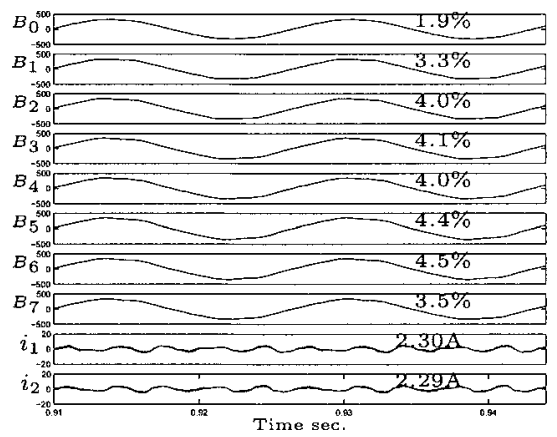

(e) Bus voltages and AFUs currents in Fig. 13(b)

Fig. 13. Simulation circuits and results in the loop line

can reduce the voltage distortion throughout the line more effectively if AFUs are deployed in the distributed fashion. In the case of harmonic termination approach, in which AFUs are installed in a concentrated fashion, the voltage distortion near bus 2 and bus 3 can not be improved, or may become worse, with increased AFU capacities due to the "whack-amole" phenomenon as shown in Fig. 12(a). Therefore, the harmonic damping effectiveness of distributed deployment of active filters is improved compared to the concentrated deployment of harmonic termination active filter.
Different allocations of AFUs are also simulated, and THDv on all buses for each allocation are listed in Table 2. These results indicate that the installation location of $\mathrm{AFU}_{2}$ have great impact on the filtering capabilities of the DAFS. The analysis discussed in Sec. 3.1 suggests that the optimal location for $A F U_{2}$ is at bus 2. Table 2 shows that installating $A F U_{2}$ at bus 2 or at bus 3 produces similar results. This is due to the slight difference between distributed-parameter model and lumped circuit model for the radial line.

4.2 Loop Line Both the concentrated deployment 


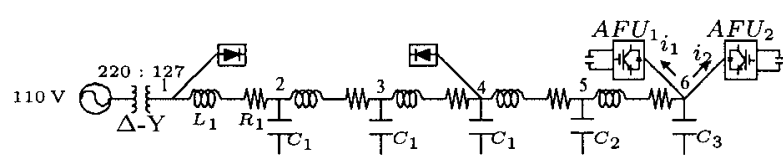

(a) $A F U_{1}, A F U_{2}$ at bus 6

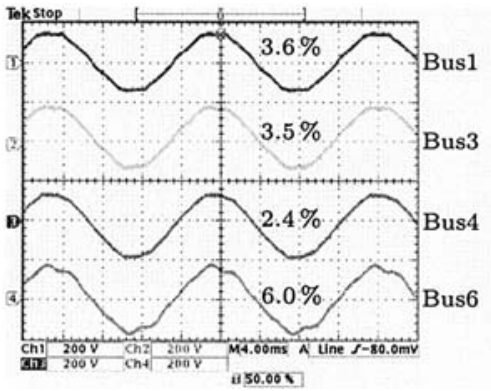

(c) Bus voltages when AFUs are off

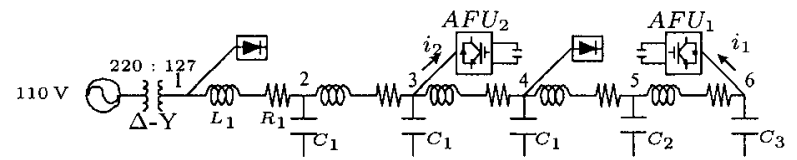

(b) $A F U_{1}$ at bus $6, A F U_{2}$ at bus 3

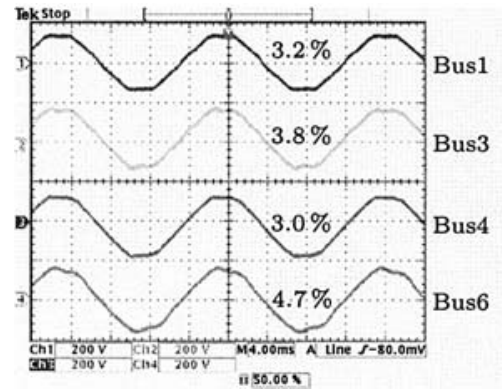

(d) Bus voltages in Fig. 14(a)

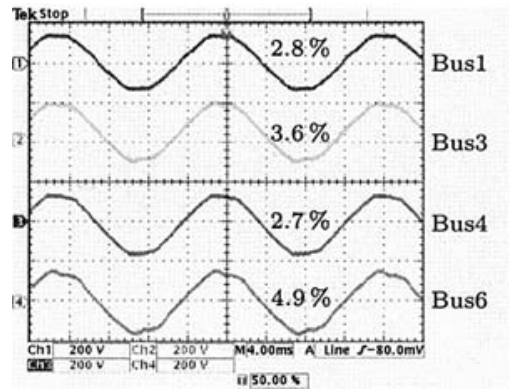

(e) Bus voltages in Fig. 14(b)

Fig. 14. Experimental circuits and results in the radial line

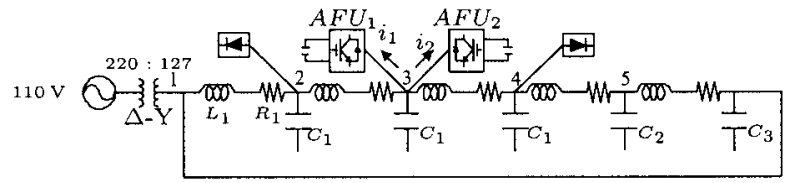

(a) $A F U_{1}, A F U_{2}$ at bus 3

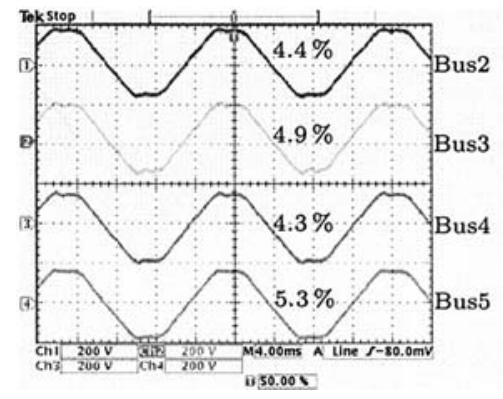

(c) Bus voltages when AFUs are off

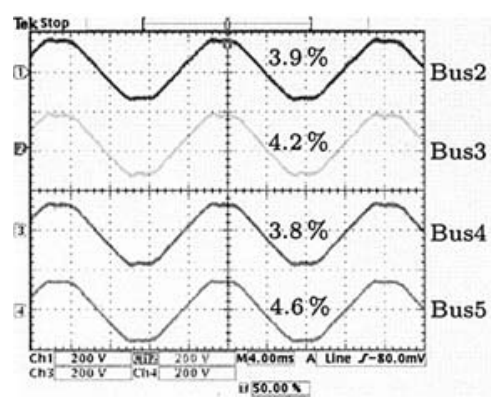

(d) Bus voltages in Fig. 15(a)

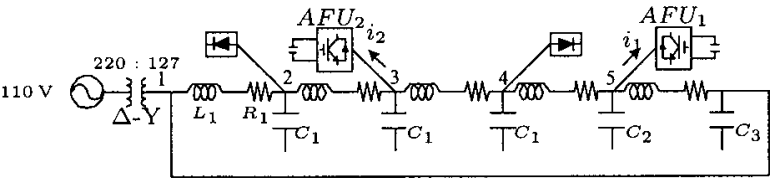

(b) $A F U_{1}$ at bus $5, A F U_{2}$ at bus 3

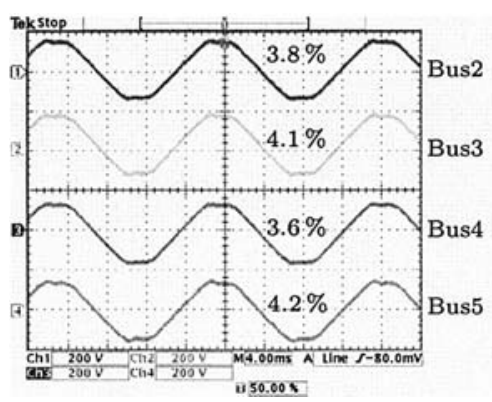

(e) Bus voltages in Fig. 15(b)

Fig. 15. Experimental circuits and results in the loop line

and the distributed deployment of AFUs in the loop line are tested to verify the harmonic damping effectiveness.

Fig. 13(a) shows the circuit model of the loop line. Both $A F U_{1}$ and $A F U_{2}$ are rated at $1.0 \mathrm{kVA}$, and installed at bus 5 to examine the potency of concentrated deployment of active filters. Before the AFUs are started, the voltages along the line are severely distorted, especially around bus 5, as indicated in Fig. 13(c). After the AFUs are in operation, THDv is marginally improved because the equivalent length between bus 0 and bus 5 is very closed to $\lambda_{7} / 2=4.85 \mathrm{~km}$. At steady state, both $A F U_{1}$ and $A F U_{2}$ consume $970 \mathrm{VA}$ with conductance command $0.31 \Omega^{-1}$.

In Fig. 13(b), $A F U_{1}$ is installed at bus 5 and $A F U_{2}$ is at bus 3 to verify the effectiveness of distributed deployment of DAFS. The loop circuit is divided by AFUs into three segments of approximately $3 \mathrm{~km}, 2 \mathrm{~km}$ and $3 \mathrm{~km}$. All these segments are shorter than $\lambda_{7} / 2=4.85 \mathrm{~km}$, so the seventh harmonic voltage standing wave can not be established. The suppression of THDv is much more effective than the concentrated deployment. Thanks to the $G-S$ droop characteristics, both $A F U_{1}$ and $A F U_{2}$ absorb $968 \mathrm{VA}$ of harmonic filtering workload with conductance command $G_{1}=$ $0.37 \Omega^{-1}$ and $G_{2}=0.39 \Omega^{-1}$ respectively. Compared to concentrated installation, the harmonic damping capability of distributed deployment of active filters is enhanced in the loop circuit.

\section{Experimental Results}

The test bench illustrated in Fig. 14 is constructed to verify the performance of the DAFS. The circuit parameters are given as $L_{1}=0.2 \mathrm{mH}, R_{1}=0.1 \Omega, C_{1}=150 \mu \mathrm{F}, C_{2}=$ $96 \mu \mathrm{F}, C_{3}=69 \mu \mathrm{F}$ and the volt-ampere consumption of both nonlinear loads is 320 VA. Two prototype AFUs are implemented by conventional voltage source inverters switching at $20 \mathrm{kHz}$. The droop settings of both inverters are $G_{0}=0 \Omega^{-1}$, $b_{1}=b_{2}=-1 \times 10^{-2} \mathrm{~V}^{-2}, S_{10}=S_{20}=100 \mathrm{VA}$.

5.1 Radial Line The termination installation and the distributed installation of AFUs in the radial line are evaluated. Fig. 14(a) shows the circuit arrangement of the termination installation. Both $A F U_{1}$ and $A F U_{2}$ are installed at bus 6 , and nonlinear loads are placed at bus 1 and bus 4 respectively. Before the AFUs are started, the bus voltages 

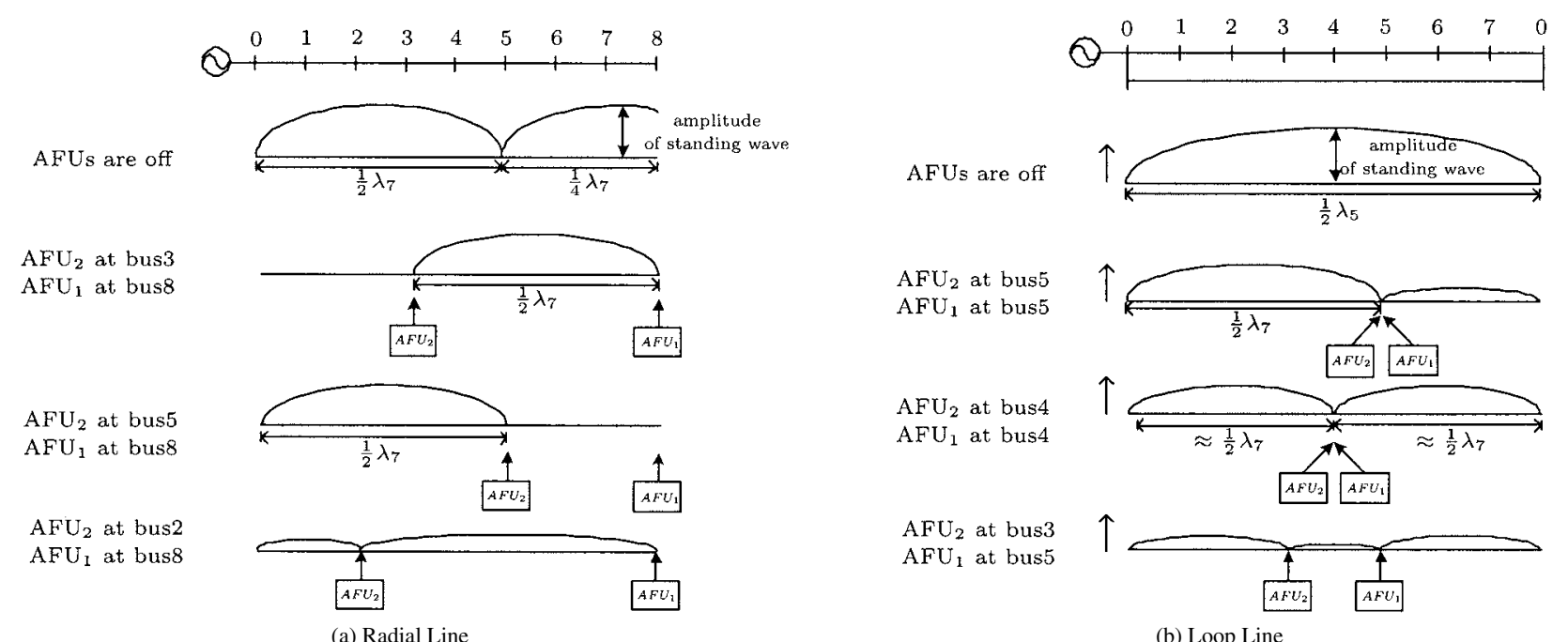

Fig. 16. Deployment strategies of the DAFS and the resulting pattern of harmonic voltages (vertical axis: the amplitude of harmonic voltage standing wave, horizontal axis: feeder location)

are severely distorted as indicated in Fig. 14(c). After the AFUs are started, Fig. 14(d) shows that the THDv reduces near bus 6 but increases at bus 3, which is resulted from the phenomenon of "whack-a-mole". At steady state, voltampere consumption and conductance commands of AFUs are $S_{1} \approx S_{2} \approx 86 \mathrm{VA}$ and $G_{1} \approx G_{2} \approx 0.14 \Omega^{-1}$. For the distributed deployment, $A F U_{1}$ and $A F U_{2}$ are installed at bus 6 and 3 respectively as illustrated in Fig. 14(b). The improvement of THDv is very significant along the entire line as shown in Fig. 14(e). At steady state, $A F U_{1}$ consumes $85 \mathrm{VA}$ and reaches $0.13 \Omega^{-1}$, while $A F U_{2}$ consumes $82 \mathrm{VA}$ at $0.16 \Omega^{-1}$. Therefore, the harmonic damping capability of the distributed deployment of AFUs is better than that of the termination deployment of AFUs in the radial line, and each AFU evenly shares the filtering workload.

5.2 Loop Line The concentration installation and the distributed installation of AFUs in the loop line are tested. Fig. 15(a) shows the concentration installation test circuit. Both $A F U_{1}$ and $A F U_{2}$ are at bus 3, and nonlinear loads are at bus 2 and bus 4 . Fig. 15(d) shows that the THDv is slightly decreased on all buses because the phenomenon of "whacka-mole" is not obvious in the short line. Both $A F U_{1}$ and $A F U_{2}$ operate at $G_{1}=G_{2}=0.11 \Omega^{-1}$ and consume $88 \mathrm{VA}$. For the distributed installation, $A F U_{1}$ and $A F U_{2}$ are installed at bus 5 and bus 3 respectively as shown in Fig. 15(b). The THDv are reduced more effectively than the concentrated deployment as illustrated in Fig. 15(e). $A F U_{1}$ consumes $90 \mathrm{VA}$ at $G_{1}=0.11 \Omega^{-1}$, while $A F U_{2}$ consumes $88 \mathrm{VA}$ at $G_{2}=0.12 \Omega^{-1}$. Therefore, the distributed deployment of AFUs in the loop line can suppress voltage harmonics effectively, and the harmonic filtering workload is also evenly shared among units.

\section{Conclusion}

This paper presents the application issues of the distributed active filter system (DAFS) in the radial distribution line and the loop distribution line. Theoretical analyses give complete explanation of the harmonic resonance and the "whack-a-mole" problem based on the harmonic voltage standing wave. The distributed-parameter model of the power line is used to evaluate the voltage harmonics and assess the harmonic damping capability of both the concentrated and the distributed deployment of AFUs.

Fig. 16 summarizes the harmonic damping performances of various AFUs deployment discussed in this paper. The vertical axis represents the amplitude of the voltage standing wave and the horizontal axis represents the locations along the line. For the radial line model in Sec. 4.1, the natural harmonic resonance occurs at the seventh harmonics. The most suitable deployment is to install $A F U_{1}$ at the end of the radial line for harmonics termination, and $A F U_{2}$ at bus 2 to damp the crest of seventh harmonics. In the loop line model in Sec. 4.2, the natural harmonic resonance occurs at the fifth harmonics. The suitable deployment is to install $A F U_{1}$ and $A F U_{2}$ at bus 5 and bus 3 respectively. The key to deploying AFUs is to break the line into several segments, and the length of each segment should be shorter than half wavelength of dominant harmonics for most effective damping.

Both the harmonic termination deployment of AFUs in the radial line and the concentrated deployment of AFUs in the loop line may result in the "whack-a-mole" phenomenon, and the voltage distortion in certain locations along the line may degrade. The proposed distributed deployment of AFUs provides adaquate harmonic damping along the line, and hence reduces the voltage distortion comprehensively. In addition, the proposed $G-S$ droop control method of the DAFS can coordinate multiple active filter units to share the harmonic filtering workload evenly without any communications. Compared to a large-capacity active filter installed at a certain location within the power system, the DAFS offers great filtering capability by integrating several small-capacity highbandwidth active filter units installed at various locations, and provides superior scalability and flexibility to cope with the growth of harmonic producing loads within the power system.

(Manuscript received May 10, 2005,

revised Dec. 2, 2005) 


\section{References}

( 1 ) H. Akagi: "Control strategy and site selection of a shunt active filter for damping of harmonic propagation in power distribution system", IEEE Trans. Power Delivery, Vol.12, No.2, pp.354-363 (1997-1)

( 2 ) H. Akagi, H. Fujita, and K. Wada: "A shunt active filter based on voltage detection for harmonic termination of a radial power distribution line", IEEE Trans. Ind. Applicat., pp.638-645 (1999-5/6)

( 3 ) K. Wada, H. Fujita, and H. Akagi: "Considerations of a shunt active filter based on voltage detection for installation on a long distribution feeder", IEEE Trans. Ind. Applicat., pp.1123-1130 (2002-7/8)

( 4 ) P. Jintakosonwit, H. Fujita, H. Akagi, and S. Ogasawara: "Implementation and performance of cooperative control of shunt active filters for harmonic damping throughout a power distribution system", IEEE Trans. Ind. Appl., Vol.39, No.2, pp.556-564 (2003-3/4)

( 5 ) D.K. Cheng: Field and Wave Electromagnetics, Reading, MA: AddisonWesley (1989)

( 6 ) P.T. Cheng and Z.L. Lee: "Distributed active filter systems (dafs): A new approach to power system harmonics", IEEE IAS 39th Annual Meeting, pp.94101 (2004)

( 7 ) M. Saito, T. Takeshita, and N. Matsui: "Modeling and harmonic suppression for power distribution system", IEEE Trans. Ind. Electron., Vol.50, No.6, pp.1148-1158 (2003-12)

\section{Appendix}

\section{Parameter Definitions in Fig. 1}

$z$ : the characteristic impedance of the line $z_{1}$ : the impedance at $A F U_{2}$ excluding $G_{2}$ $z_{2}$ : the impedance at $A F U_{2}$ including $G_{2}$

$$
z_{1}=z \frac{\frac{1}{G_{1}}+z \tanh \gamma_{h} l_{2}}{z+\frac{1}{G_{1}} \tanh \gamma_{h} l_{2}}, \quad z_{2}=\frac{\frac{1}{G_{2}} \cdot z_{1}}{\frac{1}{G_{2}}+z_{1}}
$$

\section{Parameter definitions in Fig. 3}

$z_{11}$ : the impedance at $A F U_{1}$ excluding $G_{1}$ in $\mathrm{x}$ axis $z_{12}$ : the impedance at $A F U_{1}$ including $G_{1}$ in $\mathrm{x}$ axis $z_{13}$ : the impedance at $A F U_{2}$ excluding $G_{2}$ in $\mathrm{x}$ axis $z_{14}$ : the impedance at $A F U_{2}$ including $G_{2}$ in $\mathrm{x}$ axis $z_{21}$ : the impedance at $A F U_{2}$ excluding $G_{2}$ in y axis $z_{22}$ : the impedance at $A F U_{2}$ including $G_{2}$ in y axis $z_{23}$ : the impedance at $A F U_{1}$ excluding $G_{1}$ in y axis $z_{24}$ : the impedance at $A F U_{1}$ including $G_{1}$ in y axis

$$
\begin{array}{ll}
z_{11}=z \tanh \gamma_{h} l_{3}, & z_{12}=\frac{\frac{1}{G_{1}} \cdot z_{11}}{\frac{1}{G_{1}}+z_{11}} \\
z_{13}=z \frac{z_{12}+z \tanh \gamma_{h} l_{2}}{z+z_{12} \tanh \gamma_{h} l_{2}}, & z_{14}=\frac{\frac{1}{G_{2}} \cdot z_{13}}{\frac{1}{G_{2}}+z_{13}} \\
z_{21}=z \tanh \gamma_{h} l_{1}, & z_{22}=\frac{\frac{1}{G_{2}} \cdot z_{21}}{\frac{1}{G_{2}}+z_{21}} \\
z_{23}=z \frac{z_{22}+z \tanh \gamma_{h} l_{2}}{z+z_{22} \tanh \gamma_{h} l_{2}}, & z_{24}=\frac{\frac{1}{G_{1}} \cdot z_{23}}{\frac{1}{G_{1}}+z_{23}}
\end{array}
$$

Po-Tai Cheng (Non-member) received the B.S. degree from National

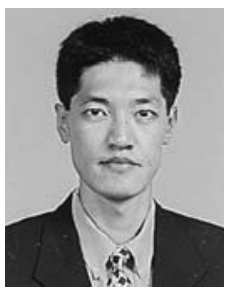
Chiao Tung University, Hsinchu, Taiwan in 1990 and M.S.E.E. and Ph.D. degrees from the University of Wisconsin, Madison, WI, USA in 1994 and 1999 respectively. $\mathrm{He}$ is currently an Associate Professor in the Department of Electrical Engineering, National Tsing Hua University, Hsinchu, Taiwan. His research interests include active filters, utility applications of power electronics, power quality issues and high power converters.

Tzung-Lin Lee (Non-member) was born in Chiayi, Taiwan in 1970.

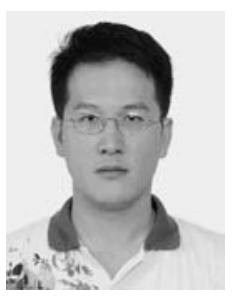
He received the B.S. degree from Chung Yuan Christian University, Taoyuan, Taiwan, in 1993, and the M.S. degree from National Chung Cheng University, Chiayi, Taiwan, in 1995, both in electrical engineering. He is working toward Ph.D. degree in the Department of Electrical Engineering, National Tsing Hua University, Taiwan. From 1997 to 2001, he was a research engineer at the Microwave Department of Industrial Technology Research Institute, Hsinchu, Taiwan. His research interests include active power filter and high-power converter application. 